\title{
Determining the Redshift of Reionization from the Spectra of High\#Redshift Sources
}

\section{Citation}

Haiman, Zoltan, and Abraham Loeb. 1999. "Determining the Redshift of Reionization from the Spectra of High\#Redshift Sources." The Astrophysical Journal 519 (2): 479-85. https:// doi.org/10.1086/307393.

\section{Permanent link}

http://nrs.harvard.edu/urn-3:HUL.InstRepos:41393244

\section{Terms of Use}

This article was downloaded from Harvard University's DASH repository, and is made available under the terms and conditions applicable to Other Posted Material, as set forth at http:// nrs.harvard.edu/urn-3:HUL.InstRepos:dash.current.terms-of-use\#LAA

\section{Share Your Story}

The Harvard community has made this article openly available.

Please share how this access benefits you. Submit a story.

Accessibility 
THE AstrophysicAL JoURNAL, 519:479-485, 1999 July 10

(c) 1999. The American Astronomical Society. All rights reserved. Printed in U.S.A.

\title{
DETERMINING THE REDSHIFT OF REIONIZATION FROM THE SPECTRA OF HIGH-REDSHIFT SOURCES \\ ZOLTÁN HAIMAN $^{1,2}$ AND ABRAHAM LOEB ${ }^{1}$ \\ Received 1998 July 8; accepted 1999 February 15
}

\begin{abstract}
The redshift at which the universe was reionized is currently unknown. We examine the optimal strategy for extracting this redshift, $z_{\text {reion }}$, from the spectra of early sources. For a source located at a redshift $z_{\mathrm{s}}$ beyond but close to the reionization redshift, $\left(1+z_{\text {reion }}\right)<\left(1+z_{\mathrm{s}}\right)<32 / 27\left(1+z_{\text {reion }}\right)$, the GunnPeterson trough splits into disjoint $\mathrm{Ly} \alpha, \mathrm{Ly} \beta$, and possibly higher Lyman series troughs, with some transmitted flux between these troughs. We show that although the transmitted flux is suppressed considerably by the dense Ly $\alpha$ forest at high redshifts, it could still be detectable as long as $z_{\text {reion }} \lesssim 8$. If the ionizing sources are bright and have a short duty cycle (e.g., if they are miniquasars or starbursts with a lifetime $\lesssim 10^{6} \mathrm{yr}$ ), then breakthrough occurs shortly after the $\mathrm{H}$ II regions around them overlap, and the transmitted flux provides a measurement of $z_{\text {reion }}$. The Next Generation Space Telescope will reach the spectroscopic sensitivity required for such a measurement.
\end{abstract}

Subject headings: cosmology: theory — galaxies: formation — intergalactic medium quasars: absorption lines - radiative transfer

\section{INTRODUCTION}

The standard big bang model predicts that the primeval plasma recombined and became predominantly neutral as the universe cooled below a temperature of several thousand degrees at a redshift $z \sim 10^{3}$ (Peebles 1968). Indeed, the recent detection of cosmic microwave background (CMB) anisotropies rules out a fully ionized intergalactic medium (IGM) beyond $z \sim 300$ (Scott, Silk, \& White 1995). However, the lack of a Gunn-Peterson (GP) trough (Gunn \& Peterson 1965) in the spectra of high-redshift quasars (Schneider, Schmidt, \& Gunn 1991) and galaxies (Franx et al. 1997) implies that the IGM is highly ionized at low redshifts, $z \lesssim 5$. Taken together, these two observations indicate that the IGM was reionized in the redshift interval $5 \lesssim z_{\text {reion }} \lesssim 300$. The epoch of reionization marks the end of the "dark ages" during which the cosmic radiation background was dominated by the ever-fading CMB at all wavelengths. During this epoch, ionized hydrogen (H II) again started to occupy most of the volume of the universe. Most likely, the phase transition from $\mathrm{H}$ I to $\mathrm{H}$ II was triggered by the emission from the first stars and miniquasars in the universe (see Loeb 1998 and references therein).

The characteristic distance between the sources that ionized the universe was much smaller than the Hubble scale, and the overlap of the $\mathrm{H}$ II regions surrounding these sources was completed on a timescale much shorter than the Hubble time. The resulting reionization redshift, $z_{\text {reion }}$, is therefore sharply defined and serves as an important milestone in the thermal history of the universe. Measurement of this parameter would also constrain the small-scale power of primordial density fluctuations that produced the first objects. Theoretical models and three-dimensional simulations predict that reionization occurs at $7 \lesssim z_{\text {reion }} \lesssim$ 20 , just beyond the horizon of current observations (Haiman \& Loeb 1997, 1998; Gnedin \& Ostriker 1997). Forthcoming instruments, such as the Space Infrared

\footnotetext{
${ }^{1}$ Astronomy Department, Harvard University, 60 Garden Street, Cambridge, MA 02138.

2 NASA/Fermilab Astrophysics Center, Fermi National Accelerator Laboratory, P.O. Box 500, Batavia, IL 60510.
}

Telescope Facility (SIRTF) and the Next Generation Space Telescope (NGST) are expected to reach the sensitivity required for the detection of sources beyond the reionization redshift. It is therefore timely to ask: how could one infer $z_{\text {reion }}$ from the observed spectra of these sources?

The spectrum of a source at a redshift $z_{\mathrm{s}}>z_{\text {reion }}$ should show a GP trough due to absorption by the neutral IGM at wavelengths shorter than the local Ly $\alpha$ resonance at the source, $\lambda_{\text {obs }}<\lambda_{\alpha}\left(1+z_{\mathrm{s}}\right)$. By itself, the detection of such a trough would not uniquely establish the fact that the source is located beyond $z_{\text {reion }}$, since the lack of any observed flux could be equally caused by (1) ionized regions with some residual neutral fraction, (2) individual damped Ly $\alpha$ absorbers, or (3) line blanketing from lower column density Ly $\alpha$ forest absorbers (see, e.g., the spectrum of a $z=5.34$ galaxy taken by Dey et al. 1998). An alternative approach proposed by Miralda-Escudé (1998) is to study the detailed shape of the damping wing of the GP trough. Although a measurement of this shape could ideally determine the optical depth of the absorbing $\mathrm{H}$ I slab between the source redshift and reionization, it is also likely to be compromised by contaminating effects such as (1) redshift distortions due to peculiar velocities, (2) uncertainties in the absorption profile due to the unknown intrinsic shape of the Ly $\alpha$ emission line from the source, (3) ionization of the IGM in the vicinity of the source due to the source emission, and (4) the existence of a damped $\operatorname{Ly} \alpha$ absorber outside or inside the source.

In this paper we consider a different approach for measuring $z_{\text {reion }}$. Our method relies on the existence of some transmitted flux between the $\operatorname{Ly} \alpha$ and $\operatorname{Ly} \beta$ absorption troughs in the spectra of sources that are located just beyond the reionization redshift. For such sources, the GP troughs due to different Lyman series resonances do not necessarily overlap, and the transmitted flux between these troughs is affected only by the post-reionization $\operatorname{Ly} \alpha$ forest. In $\S 2$, we simulate the absorption by the dense Ly $\alpha$ forest (or "Ly $\alpha$ jungle") and demonstrate that the transmitted flux features are detectable. In $\S 3$, we estimate the number of sources that are sufficiently bright to allow detection of 
these features with NGST. Finally, $₫ 4$ summarizes the main conclusions of this work.

\section{SPECTRA OF HIGH-REDSHIFT SOURCES}

The phase transition from $\mathrm{H}$ I to $\mathrm{H}$ iI occurs almost simultaneously throughout the IGM volume when the expanding $\mathrm{H}$ II regions around the separate ionizing sources overlap (Arons \& Wingert 1972). The expansion of spherical ionization fronts around steady sources can be calculated analytically (Shapiro \& Giroux 1987). The process of overlap is nearly instantaneous (Haiman \& Loeb 1998, hereafter HL98) and defines a corresponding redshift, $z_{\text {overlap }}$. However, at $z_{\text {overlap }}$ each individual $\mathrm{H}$ in region could still have a nonnegligible $\operatorname{Ly} \alpha$ optical depth due to its residual H I fraction (see Shapiro et al. 1987). The GP trough from the uniform IGM disappears only at a somewhat later redshift, $z_{\text {reion }}$, when the average Ly $\alpha$ optical depth of the smooth IGM drops below unity (we distinguish between this GP absorption and the absorption produced as a result of IGM clumpiness by the post-reionization Ly $\alpha$ forest). In this paper, we assume that the delay between $z_{\text {overlap }}$ and $z_{\text {reion }}$ is small and set $z_{\text {overlap }}=z_{\text {reion }}$, based on the following reasoning.

The Ly $\alpha$ optical depth across a stationary H II sphere is as large as $\sim 10^{4}$ (Osterbrock 1974), but cosmological H II regions are much thinner because of the cosmological redshifting of the Ly $\alpha$ photons away from resonance. For concreteness, consider a case in which the $\mathrm{H}$ II regions are driven by miniquasars in cold dark matter halos of a virial temperature $\gtrsim 10^{4} \quad \mathrm{~K} \quad\left(M_{\text {halo }} \gtrsim 1.5 \times 10^{8} \quad M_{\odot}\right)$. This minimum halo size is dictated by the requirement that its gas would be able to cool efficiently via atomic transitions. With black hole to halo mass ratios $M_{\mathrm{bh}} / M_{\text {halo }} \sim 10^{-3}$ and quasar lifetimes $t_{\mathrm{Q}} \sim 10^{6} \mathrm{yr}$, the typical proper radius of an $\mathrm{H}$ II region is $R_{\mathrm{H} \text { II }} \sim 0.05 \mathrm{Mpc}$ (see HL98 for details). The short-lived miniquasars have a small duty cycle, and when $\mathrm{H}$ II regions around two such miniquasars first overlap, the neutral fraction at their intersection is $x \approx 2 \times 10^{-5}$ in photoionization equilibrium. Because of the short duty cycle of the ionizing sources, the ionizing miniquasar flux at the time of overlap is not far below the eventual flux of the cosmic UV background, integrated over a Hubble time. The resulting IGM optical depth at the time of overlap is $\tau \sim 3$. The GP trough disappears as soon as the neutral fraction is reduced by another factor of $\sim 3$, i.e., when the local UV flux is increased by this factor. Such an increase could occur shortly after the overlap epoch, since it requires the cumulative contribution from only a few additional sources. To encounter a sufficient number of already existing active sources, the required radius is $\sim 100 \times 3 \times 0.05 \mathrm{Mpc}=15$ $\mathrm{Mpc}$ (where the factor of $\sim 100$ is the inverse of the duty cycle). The light crossing time across this radius causes a short $(\sim 5 \%)$ delay for the breakthrough redshift, $z_{\text {reion }}$, relative to the overlap redshift, with $\left(z_{\text {overlap }}-z_{\text {reion }}\right) \approx 0.4$ in this case. Note, however, that clustering of the ionizing sources or the appearance of new active sources on a timescale significantly shorter than the local Hubble time can shorten this redshift delay. On the other hand, if the ionizing sources have a significantly longer lifetime than we assumed (e.g., if they are long-lived star-forming galaxies), then reionization would be more gradual, as outlined by Miralda-Escudé (1998). We illustrate this possibility in Figure 4 below. In this case, we point out that the $\operatorname{Ly} \beta$ optical depth is $\sim 3.5$ times below that of Ly $\alpha$, so that the
Ly $\beta$ and higher GP troughs are more sharply defined than the Ly $\alpha$ trough for gradual reionization.

We now consider the spectrum of a source located at a redshift $z_{\mathrm{s}}>z_{\text {reion }}$. Ly $\alpha$ absorption by neutral hydrogen between $z_{\text {reion }}$ and $z_{\mathrm{s}}$ suppresses the flux in the observed wavelength interval, $\left(1+z_{\text {reion }}\right) \lambda_{\alpha}<\lambda_{\text {obs }}<\left(1+z_{\mathrm{s}}\right) \lambda_{\alpha}$; Ly $\beta$ absorption causes a second trough at $\left(1+z_{\text {reion }}\right) \lambda_{\beta}<\lambda_{\text {obs }}<$ $\left(1+z_{\mathrm{s}}\right) \lambda_{\beta}$, and higher Lyman series lines produce analogous troughs at shorter wavelengths. In the absence of other effects, the resulting spectrum would consist of a generic sequence of troughs separated by blocks of transmitted flux, as depicted in Figure 1. In this illustration we assumed that reionization occurred suddenly as discussed above, at $z_{\text {reion }}=7$, but included the damping wings of the Lyman lines at both edges of the absorption troughs (see the Appendix in Miralda-Escudé 1998). Note that a Ly $\alpha$ photon emitted by the source can escape from its host's $\mathrm{H}$ II region and travel across the uniform IGM only if the $\mathrm{H}$ II region has an unusually large radius of at least $\sim 1 \mathrm{Mpc}$. The emitted Ly $\alpha$ photons are much more likely to be absorbed by the IGM. Below we consider observations on a telescope with an angular resolution sufficiently high that the diffuse Ly $\alpha$ reemission by the IGM can be ignored.

The top panel of Figure 1 shows that the closer $z_{s}$ gets to $z_{\text {reion }}$, the narrower the GP troughs become and the more similar they look to individual Ly $\alpha$ absorbers. Equation (1) below predicts a few damped Ly $\alpha$ systems with neutral hydrogen column densities $N_{\mathrm{H}} \gtrsim 10^{20} \mathrm{~cm}^{-2}$ in the spectra of a source at redshifts $z_{\mathrm{s}} \gtrsim 5$. A single absorber with $N_{\mathrm{H}}=$ $10^{20} \mathrm{~cm}^{-2}$ and $b=35 \mathrm{~km} \mathrm{~s}^{-1}$ would have a rest-frame equivalent width of $7.7 \AA$. In order to avoid confusion with such an absorber, the width of the GP troughs must be $\gtrsim 8\left(1+z_{\mathrm{s}}\right) \AA$. Note also that peculiar velocities could shift the location of the narrow troughs by $\sim 10 \AA$. The bottom

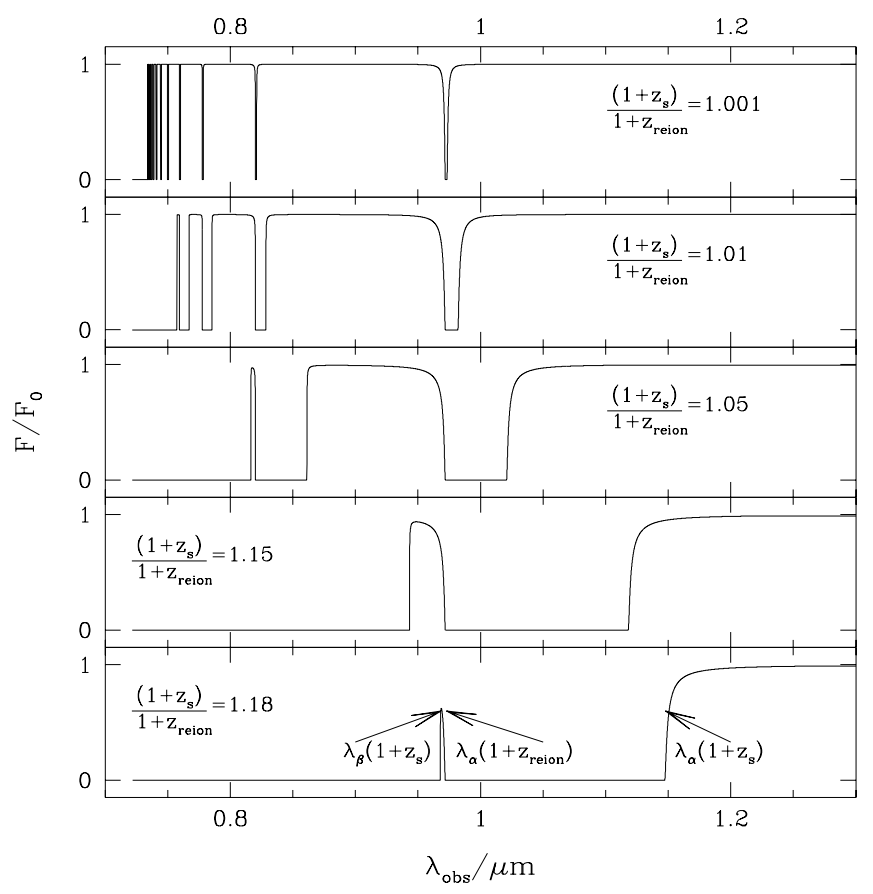

FIG. 1.-Spectra of five sources located beyond the reionization redshift (here taken to be $z_{\text {reion }}=7$ ), when all contaminating effects, such as peculiar velocities, the ionization of the IGM in the vicinity of the source, or absorption by the Ly $\alpha$ forest, are ignored. 
panel of Figure 1 also indicates that as the source redshift approaches the limiting value $\left(1+z_{\mathrm{s}}\right) /\left(1+z_{\text {reion }}\right)=\lambda_{\beta} / \lambda_{\alpha}=$ $32 / 27$, only a single narrow block of transmitted flux remains between the $\operatorname{Ly} \alpha$ and $\operatorname{Ly} \beta$ troughs. At still higher redshifts this window disappears, leaving a continuous GP trough at wavelengths $\lambda_{\text {obs }}<\lambda_{\alpha}\left(1+z_{\text {reion }}\right)$ that carries no information about the reionization redshift.

Reionization also leaves a distinct mark in the soft X-ray regime. The source spectrum would be strongly suppressed at the Lyman edge, $\lambda_{\text {obs }}=912\left(1+z_{\mathrm{s}}\right) \AA$, owing to the large continuum optical depth in from $\mathrm{H}$ and He ionizations $\left(\tau_{\text {cont }} \sim 10^{5}\right)$. The optical depth drops at shorter wavelengths owing to the sharp decline in the ionization cross sections, $\sigma \propto \lambda^{3}$. The flux recovers its intrinsic amplitude at $E \gtrsim 0.1 \mathrm{keV}$, where the optical depth falls below unity. The observed photon energy where this occurs provides a measure of the reionization redshift, as long as the value of $\Omega_{\mathrm{b}} h^{2}$ is independently measured. Note that the hydrogen GP signal could be contaminated by absorption from intervening Lyman limit and damped Ly $\alpha$ systems, the equivalent tail of the He II GP trough, and Galactic foreground absorption (O'Flaherty \& Jakobsen 1997). Ignoring these issues, it is interesting to ask whether $A X A F$ would be able to detect the spectral signature in the ideal case. For a source whose observed flux at $E=0.1 \mathrm{keV}$ is $100 \mathrm{nJy}$, the $A X A F$ count rate in the $0.08-0.15 \mathrm{keV}$ band would be $10^{-3}$ $\mathrm{s}^{-1}$, sufficiently large to collect 100 photons in $10^{5} \mathrm{s.}^{3}$ The ability of $A X A F$ to determine $z_{\text {reion }}$ relies on the existence of sufficiently bright sources beyond the reionization redshift. In the case where these sources are early miniquasars, their required infrared flux is $\gtrsim 10 \mu \mathrm{Jy}$. As illustrated by Figure 6 below, such sources are expected to be very rare at $z>5$.

The spectra shown in Figure 1 are highly idealized in that they take account only of absorption by the homogeneous $\mathrm{H}$ I gas between $z_{s}$ and $z_{\text {reion }}$. In reality, the blocks of transmitted flux shown in Figure 1 will be suppressed because of absorption by the residual $\mathrm{H} \mathrm{I}$ islands in the clumped IGM, which produce the Ly $\alpha$ forest after reionization. In order to simulate the effect of the dense Ly $\alpha$ forest (or Ly $\alpha$ jungle), we created mock catalogs of absorption lines with statistical properties constrained by observational data at $z<5$. Fardal, Giroux, \& Shull (1998) have recently analyzed in detail the statistics of Ly $\alpha$ absorbers observed between redshifts $1.5<z<4.5$. Using a maximum likelihood method, they obtained the mean number $n\left(N_{\mathrm{H}}, b, z\right) d N_{\mathrm{H}} d b d z$ of clouds with $\mathrm{H}$ I column density between $N_{\mathrm{H}}$ and $N_{\mathrm{H}}+d N_{\mathrm{H}}$, Doppler velocity parameter between $b$ and $b+d b$, and redshift between $z$ and $z+d z$. In their procedure, the Doppler parameter $b$ and column density $N_{\mathrm{H}}$ is obtained for each absorber by Voigt profile fitting. Their resulting best fit for $n\left(N_{\mathrm{H}}, b, z\right)$ is a four-segment broken power law in $N_{\mathrm{H}}$, where each segment is valid for a different range of values for $N_{\mathrm{H}}$ and has the form

$$
n\left(N_{\mathrm{H}}, b, z\right)=A(1+z)^{\gamma}\left(\frac{N_{\mathrm{H}}}{10^{17} \mathrm{~cm}^{-2}}\right)^{-\beta} p(b) \mathrm{cm}^{2} \mathrm{~km}^{-1} \mathrm{~s},
$$

\footnotetext{
${ }^{3}$ We consider detection by the High-Resolution Camera and the LowEnergy Transmission Grating, based on the proposal planning tool kit at http://asc.harvard.edu/cgi-bin/prop_toolkit.cgi.
}

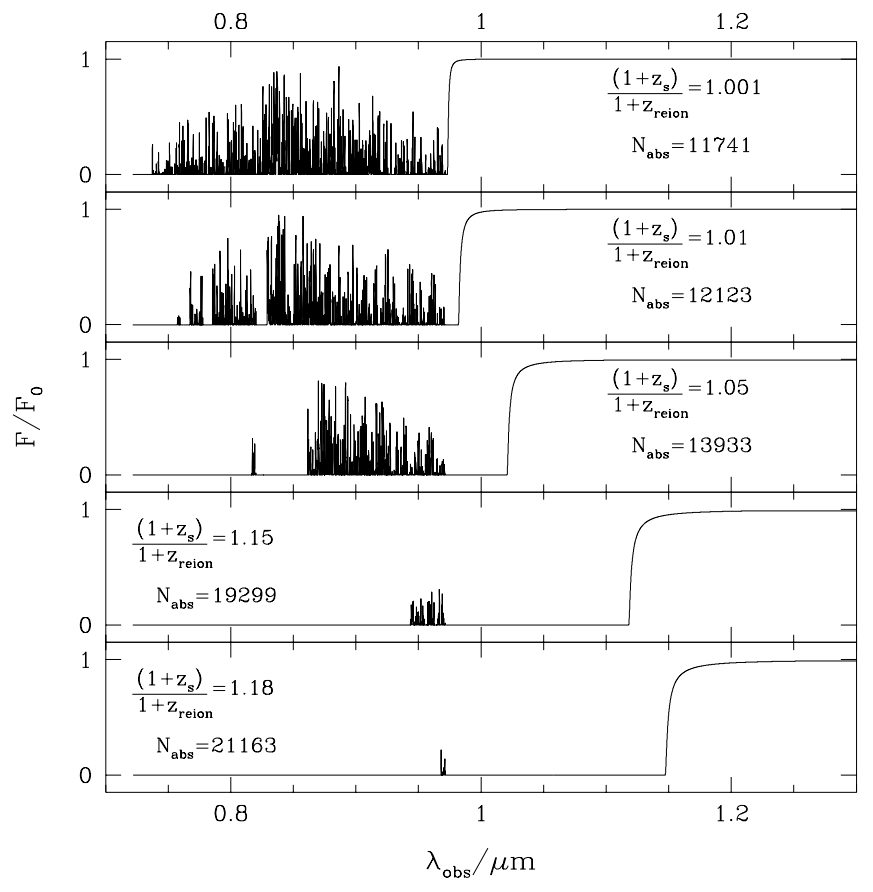

FIG. 2.- Spectra of the five sources in Fig. 1, when the absorption due to the post-reionization $\mathrm{Ly} \alpha$ forest is taken into account.

with

$$
p(b) \propto \exp \left[-\frac{\left(b-b_{0}\right)^{2}}{2 b_{*}^{2}}\right] \quad\left(b>20 \mathrm{~km} \mathrm{~s}^{-1}\right)
$$

and $\int_{0}^{\infty} p(b) d b=1, b_{0}=28 \mathrm{~km} \mathrm{~s}^{-1}$, and $b_{*}=10 \mathrm{~km} \mathrm{~s}^{-1}$. The best-fit values of $A, \beta$, and $\gamma$ for each of the four segments are summarized by Fardal, Giroux, \& Shull (1998). We used a Monte Carlo approach to generate a catalog of absorbers along the line of sight to the source, with redshifts, column densities, and $b$-parameters in the ranges $0 \leq$ $z_{i} \leq z_{\text {reion }}, 2 \times 10^{12} \mathrm{~cm}^{-2} \leq N_{\mathrm{H}, i} \leq 10^{20} \mathrm{~cm}^{-2}$, and $b_{i} \geq 0$, for $1 \leq i \leq N_{\text {abs }}=\int n d z d N_{\mathrm{H}} d b$. The probability distribution of these parameters was chosen according to the above equations. Although these equations are based on data from redshifts $1.5<z<4.5$, in the absence of additional data at higher $z$, we extend their validity to all redshifts below $z_{\text {reion }}$. A more rigorous extrapolation of these equations to high redshift would be to use three-dimensional numerical simulations of the high-redshift IGM (e.g., Hernquist et al. 1996). However, even such a calculation would be highly uncertain because of the unknown evolution of the flux and spectrum of the UV background at $z>5$.

In computing the observed flux $F$ from the source at a wavelength $\lambda_{\text {obs }}$ we include absorption by the first nine Lyman series lines from each of the $N_{\text {abs }}\left(z_{\text {reion }}\right)$ absorbers along the line of sight,

$$
F\left(\lambda_{\mathrm{obs}}\right)=F_{0} \prod_{n \in[1,9]} \prod_{i \in\left[1, N_{\mathrm{abs}}\right]} e^{-\tau_{i, n} U\left(x_{i, n}, a_{i, n}\right)} .
$$

Here $F_{0}$ is the continuum flux that would have been observed in the absence of Ly $\alpha$ absorbers; $\tau_{i, n}=$ $\left(\pi e^{2} / m_{e} c\right) N_{\mathrm{H}, i} \lambda_{n} f_{n} / b_{i}$ is the integrated optical depth for the $n$th Lyman line of the $i$ th absorber; and $\lambda_{n}$ and $f_{n}$ are the rest wavelength and oscillator strength of this line. The profile of each line is given by the normalized Voigt func- 
tion $U(x, a)$, where $x_{i, n} \equiv\left(c / b_{i}\right)\left[\lambda_{\text {obs }} /\left(1+z_{i}\right)-\lambda_{n}\right] / \lambda_{n} ; a_{i, n} \equiv$ $\Gamma_{n} \lambda_{n} / 4 \pi b i$; and $\Gamma_{n}$ is the natural decay rate of the $n$th Lyman line (see Press \& Rybicki 1993 for more details).

Figure 2 shows five examples of source spectra processed through the mock Ly $\alpha$ jungle. As in Figure 1, we assume sudden reionization at $z_{\text {reion }}=7$ but include the damping wings of the GP troughs. The several thousand absorbers along a typical line of sight yield a considerable mean optical depth, $\tau \approx 3.4$. Although the underlying continuum flux is not visible at any wavelength, the figure reveals a series of narrow features of transmitted flux - remnants of the processing by the underlying Ly $\alpha$ jungle - to which we refer hereafter as "transmission features." The spectra contain many such features with central flux values that rise infrequently up to a significant fraction (up to $\sim 50 \%$ ) of the original continuum flux. These features could be detected by an instrument with a sufficiently high sensitivity and spectral resolution.

As an example, we show in Figure 3 a blowup of the spectrum around the $\operatorname{Ly} \alpha$ and $\operatorname{Ly} \beta$ GP troughs of a source with a redshift $z_{\mathrm{s}}=7.08$. The spectrum indeed contains numerous transmission features; these features are typically a few A wide, have a central intensity of a few percent of the underlying continuum, and are separated by $\sim 5 \AA$. With a sensitivity reaching $1 \%$ of the continuum, the edges of the Ly $\beta$ GP trough between 8201 and $8283 \AA$ could be identified to a $\sim 5 \AA$ accuracy. A similar precision applies to the Ly $\alpha$ GP trough, although the damping wing there suppresses the flux within $\sim 10 \AA$ of the edges by a factor of $\sim 3$. The longest wavelength at which flux is detected toward the blue edge of the Ly $\beta$ GP trough, at $\sim 8201 \AA$, provides the best direct measurement of the reionization redshift. In practice, this method yields only a lower limit to $z_{\text {reion }}$, since the first observed transmission feature could be offset from the true blue edge of the GP trough. The uncertainty in the measurement of $\lambda_{\beta}\left(1+z_{\text {reion }}\right)$ would be equal to the typical separation between the transmission features. Figure 3 shows that at a sensitivity reaching $1 \%$ of the
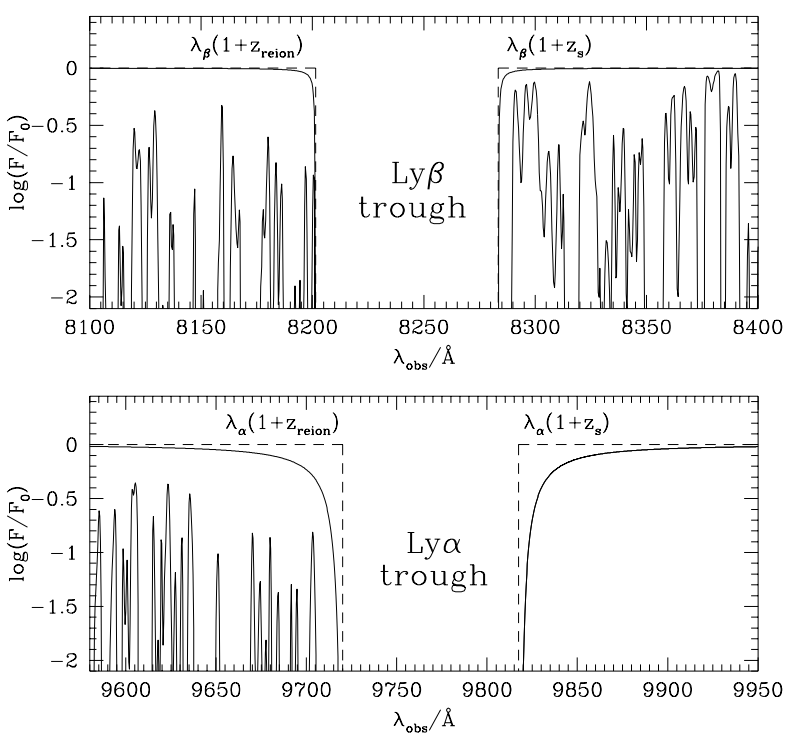

FIG. 3.-Blowup of the spectrum of a source at $z_{\mathrm{s}}=7.08$, assuming sudden reionization at a redshift $z_{\text {reion }}=7$. The solid curves show the spectrum without absorption by the high-redshift Ly $\alpha$ forest, and the dashed lines show the spectrum when the damping wings are also ignored. continuum, the separation would be small ( $\sim 5 \AA$ ), leading to a fractional error of only $\sim 5 / 8200 \sim 0.1 \%$ in the measurement of $z_{\text {reion }}$. Note that this accuracy is similar to the level allowed by peculiar velocities of a few hundred $\mathrm{km}$ $\mathrm{s}^{-1}$, which could move either observed edge of the GP trough by $\sim 10 \AA$.

It is apparent from Figure 3 that the above procedure applies only as long as the typical separation between the transmission features is much smaller than the width of the GP trough, i.e., for $\left(1+z_{\mathrm{s}}\right) \gtrsim 1.01 \times\left(1+z_{\text {reion }}\right)$. The bottom three panels in Figure 2 also show that the transmission features gradually disappear as the GP troughs of the $\operatorname{Ly} \alpha$ and $\operatorname{Ly} \beta$ resonances approach overlap at $\left(1+z_{\mathrm{s}}\right) /$ $\left(1+z_{\text {reion }}\right)=\lambda_{\alpha} / \lambda_{\beta}=32 / 27$. Hence, suitable sources must lie in the somewhat more restricted redshift range, $1.01 \lesssim$ $\left(1+z_{\mathrm{s}}\right) /\left(1+z_{\text {reion }}\right) \lesssim 1.17$. Finally, we note that an extrapolation of equation (1) to even higher redshifts predicts that the mean optical depth of the Ly $\alpha$ clouds increases strongly with redshift. For example, using our mock Ly $\alpha$ cloud catalog, we find that at wavelengths just below $\lambda_{\alpha}\left(1+z_{\text {reion }}\right)$, the mean optical depth increases from $\tau=3.4$ for $z_{\text {reion }}=7$ to $\tau=10.4$ for $z_{\text {reion }}=10$. Similar numbers $(\sim 30 \%$ higher optical depths for a less accurate description of the Ly $\alpha$ forest) are obtained by extrapolating the expressions for the mean optical depth from Madau (1995). This implies that detection of the remnant transmission features is considerably more difficult at higher redshifts, since the number density of lines above a given sensitivity threshold drops as $\sim \exp (-\tau)$. In order to detect transmission features separated by roughly $\sim 10 \AA$, as in Figure 3, the spectroscopic sensitivity must reach the approximate fractions of $\sim 10^{-1}, 10^{-2}, 10^{-3}, 10^{-4}$, and $10^{-6}$ of the continuum flux for $z_{\text {reion }}=6,7,8,9$, and 10 , respectively.

Finally, we illustrate the possibility of a more gradual reionization process in Figure 4. Rather than assuming abrupt reionization at $z_{\text {overlap }}=z_{\text {reion }}=7$, we assume that after the sudden overlap of $\mathrm{H}$ II regions at $z_{\text {overlap }}=7$, the neutral fraction within the IGM is still nonnegligible, $x=10^{-4}$. We assume further that the cosmic background
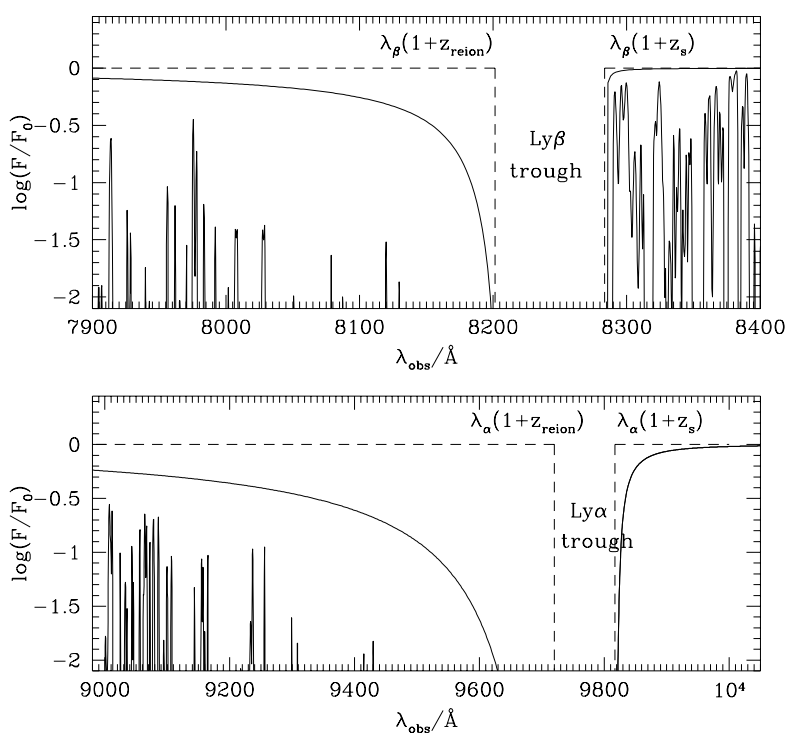

Fig. 4.- Same as Fig. 3, but assuming that the cosmic flux $J$ builds up linearly in time between $z=7$ and $z=6$, and the neutral fraction decreases as $1 / J$ from $10^{-4}$ to $10^{-6}$ during this redshift interval. 
flux $J$ increases linearly in time by a factor of 100 between $z=7$ and $z=6$, so that the neutral fraction drops (as $1 / J$ ) to $x=10^{-6}$ by $z=6$. To be consistent with this evolution of $J$, we also assume that the neutral column density of each Ly $\alpha$ cloud with $N_{\mathrm{H}}<10^{17} \mathrm{~cm}^{-2}$ (i.e., those clouds that are not self-shielding) evolves as $1 / J$ between $z=6$ and $z=7$. As Figure 4 demonstrates, the flux is suppressed by several orders of magnitude at the blue side of the GP troughs and recovers its sudden reionization value only several hundreds of $\AA$ away from the edge of the trough. Under these conditions, a measurement of the frequency where the flux appears at the blue side of the $\operatorname{Ly} \beta$ GP trough would yield a value $z \sim 6.8$, which is lower than the overlap redshift $z_{\text {overlap }}=7$. The redshift inferred from the Ly $\alpha$ trough would be still slightly lower, $z \sim 6.6$.

\section{ABUNDANCE OF SUITABLE HIGH-REDSHIFT SOURCES}

The detection of a single bright source from the epoch, $1.01\left(1+z_{\text {reion }}\right)<\left(1+z_{\mathrm{s}}\right)<1.17\left(1+z_{\text {reion }}\right)$, could in principle provide an unambiguous measurement of $z_{\text {reion }}$. How likely is it to find such sources? The best candidates are high-redshift miniquasars, since in analogy with their $z<5$ counterparts, they are expected to have a hard spectrum extending into the far-UV. Alternative sources are gammaray burst (GRB) afterglows, supernovae, or primeval galaxies. The advantage of GRB afterglows is that they are bright and have featureless power-law spectra; however, their abundance at high redshifts might be small. Supernovae and galaxies may be more abundant than either miniquasars or GRBs, but are they likely to be faint and to possess soft spectra in the relevant UV range (see, e.g., two recent detections of candidate galaxies at redshifts $z=5.64$ [Hu, Cowie, \& McMahon 1998]; and $z=5.60$ [Weymann et al. 1998]).

As the abundance of miniquasars at $z>5$ is currently unknown, we must resort to an extrapolation of the observed luminosity function (LF) of quasars at $z \lesssim 5$. Such an extrapolation has been carried out by HL98 using a simple model based on the Press-Schechter (1974) formalism. In the model of HL98, the evolution of the quasar LF at faint luminosities and high redshifts is derived from three assumptions: (1) the formation of dark-matter halos follows the Press-Schechter theory, (2) each dark halo forms a central black hole with the same efficiency, and (3) the light curve of all quasars, in Eddington units, is a universal function of time. As shown in HL98, these assumptions provide an excellent fit to the observed quasar LF at redshifts $2.6<z<4.5$ for an exponentially decaying light curve with a time-constant of $\sim 10^{6} \mathrm{yr}$. The model provides a simple and natural extrapolation of the quasar LF to high redshifts and faint luminosities. At the faint end of the LF, the number counts of miniquasars are expected to be reduced because of feedback due to photoionization heating that prevents gas from reaching the central regions of shallow potential wells. To be consistent with the lack of faint point sources in the Hubble Deep Field, we imposed a lower limit of $\sim 75 \mathrm{~km} \mathrm{~s}^{-1}$ for the circular velocities of halos harboring central black holes (Haiman, Madau, \& Loeb 1999). Figure 5 shows the resulting number counts of miniquasars, from redshifts above $z=5$ and $z=10$, as a function of the threshold flux in the 1-3.5 $\mu \mathrm{m}$ wavelength range of NGST. For reference, this figure also shows the expected number of high-redshift star clusters in a similar model in which the star formation efficiency is calibrated to the observed IGM

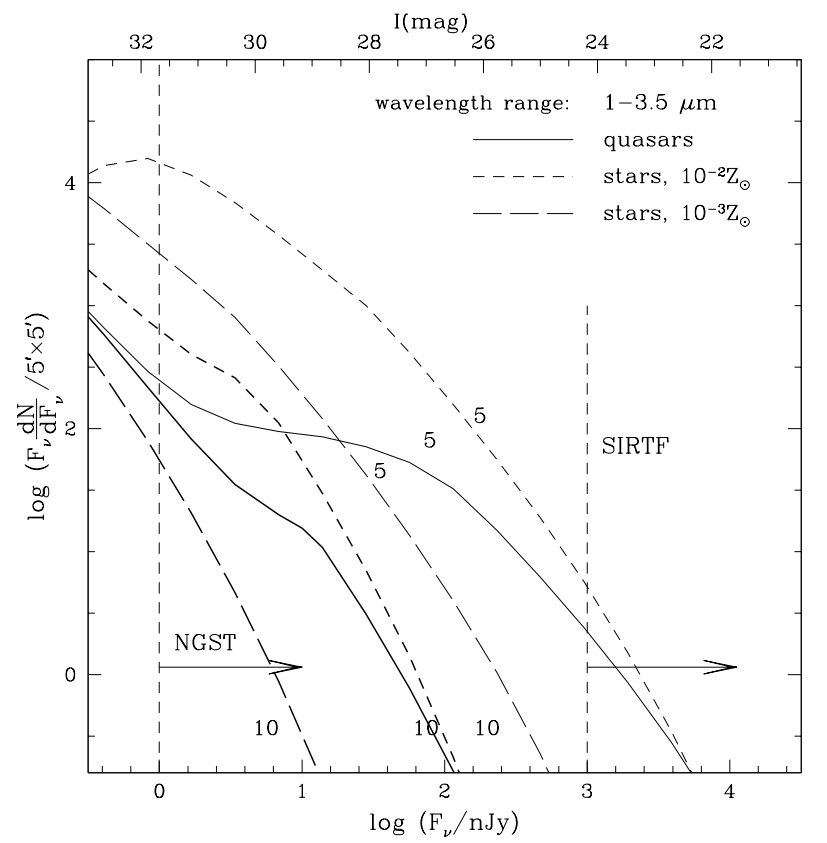

FIG. 5.-Predicted number per logarithmic flux interval of high-redshift objects that could be probed by the future space telescopes SIRTF and $N G S T$, in the wavelength range of 1-3.5 $\mu \mathrm{m}$. The observed quasar luminosity function was extrapolated to high redshifts using the model in HL98, and the star formation efficiency was calibrated to the observed IGM metallicity $\left(10^{-3} Z_{\odot} \lesssim Z \lesssim 10^{-2} Z_{\odot}\right)$ at redshift $z \sim 3$.

metallicity $\left(10^{-3} Z_{\odot} \lesssim Z \lesssim 10^{-2} Z_{\odot}\right.$; see Songaila 1997) at $z \sim 3$.

Figure 6 shows the total number $N(z, F)$ of miniquasars brighter than the minimum flux $F$ that are located within the suitable redshift interval, $1.01(1+z)<\left(1+z_{\mathrm{s}}\right)<1.17$ $(1+z)$, in a $16^{\prime} \times 16^{\prime}$ field (16 times the field of view of NGST). How many suitable sources would NGST detect? For $z_{\text {reion }}=7$, the average transmitted flux shortward of the $\operatorname{Ly} \alpha$ and $\operatorname{Ly} \beta$ GP troughs is a fraction $\exp (-\tau) \approx 1 \%$ of the continuum. Around $z \sim 7$, we expect roughly a single $\sim 10^{3}$ nJy source per $16^{\prime} \times 16^{\prime}$ field, based on Figure 6 . Such a source has an average flux of $\sim 10 \mathrm{nJy}$ blueward of $\lambda_{\alpha}\left(1+z_{\mathrm{s}}\right)$ owing to absorption by the Ly $\alpha$ jungle. According to the method depicted on Figure 3, a determination of $z_{\text {reion }}$ to $\sim 1 \%$ accuracy would require spectroscopy with a resolution of $R=100$. Requiring a signal-to-noise $(\mathrm{S} / \mathrm{N})$ ratio $=10$, the necessary integration time for a $10 \mathrm{nJy}$ source with NGST would be roughly $10 \mathrm{hr}^{4}{ }^{4}$ This is a conservative estimate for the detection of flux, since it assumes that the flux is uniformly distributed across the spectrum, while in reality it would be concentrated in transmission features that cover narrow wavelength bins and yield a higher $\mathrm{S} / \mathrm{N}$ ratio in these bins. We therefore conclude that $N G S T$ will be able to measure the reionization redshift to $\sim 1 \%$ percent accuracy up to $z_{\text {reion }}=7$. The accuracy would degrade considerably with redshift for $z_{\text {reion }} \gtrsim 7$.

The total number of sources per $16^{\prime} \times 16^{\prime}$ field, irrespective of their redshifts, is $\sim 3 \times 10^{3}, \sim 600$, and $\sim 90$ for a

\footnotetext{
${ }^{4}$ This result was obtained using the NGST Exposure Time Calculator, available at http://augusta.stsci.edu. We assumed a mirror diameter of $8 \mathrm{~m}$, and adopted the shortest wavelength currently available, $\lambda=1 \mu \mathrm{m}$.
} 


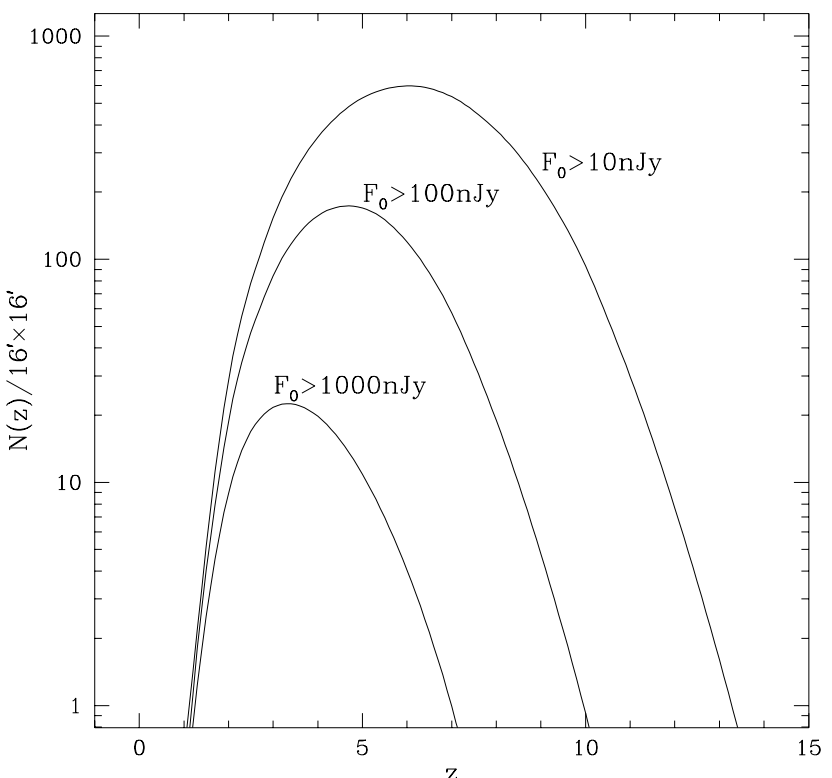

FIG. 6.-Predicted number of miniquasars within the redshift interval $1.01(1+z)<\left(1+z_{\mathrm{s}}\right)<1.17(1+z)$, with a minimum intrinsic flux $F_{0}$, in a $16^{\prime} \times 16^{\prime}$ field $\left(164^{\prime} \times 4^{\prime} N G S T\right.$ fields of view). The observed quasar luminosity function was extrapolated to high redshifts using the model in HL98.

minimum intrinsic flux of $F_{0}=10,100$, and $1000 \mathrm{nJy}$, respectively. In the most optimistic case, for which the reionization redshift is close to the peak of the relevant curve in Figure 6, up to $\sim 25 \%$ of all detected sources in each image would lie in the redshift range $1.01(1+z)<$ $\left(1+z_{\mathrm{s}}\right)<1.17(1+z)$ and would reveal the features depicted in Figure 2. However, since only a small fraction of all baryons need to be incorporated in collapsed objects in order to ionize the IGM, reionization is likely to occur before the redshift at which the source number-count peaks $\left(z_{\text {peak }}=3.5,4.5\right.$, or 6 at sensitivities of $10^{3}, 10^{2}$, or $\left.10 \mathrm{nJy}\right)$. Preselection of sources in particular redshift bins could be achieved photometrically by bracketing the associated location of their Ly $\alpha$ troughs with two narrowband filters.

\section{CONCLUSIONS}

We have shown that the Gunn-Peterson effect breaks into individual Lyman series troughs in the spectra of sources located in the redshift interval $1<\left(1+z_{\mathrm{s}}\right) /$ $\left(1+z_{\text {reion }}\right)<32 / 27$. Although the transmitted flux in between these troughs is heavily absorbed by the Ly $\alpha$ jungle after reionization, the residual features it shows allow a determination of the reionization redshift, $z_{\text {reion }}$, for sufficiently bright sources. For a single source, a reionization redshift $z_{\text {reion }} \sim 7$ could be determined to a precision of $\sim 1 \%$ with a spectroscopic sensitivity of $\sim 1 \%$ of the continuum flux. A simple model for the abundance of high- redshift miniquasars predicts that a single source per $16^{\prime} \times 16^{\prime}$ field could allow this detection at the $\sim 10 \mathrm{nJy}$ spectroscopic sensitivity of NGST. It may also be feasible to probe reionization with ground-based telescopes. Using the Keck Telescope, Dey et al. (1998) have recently detected a source at $z=5.34$ and found no continuum emission below the rest-frame Ly $\alpha$ wavelength. The authors were able to infer a lower limit of $\tau=1.2$ on the optical depth of the Ly $\alpha$ forest between 1050 and $1170 \AA$ to $z=5.34$, which falls just short of the $1.3<\tau<1.9$ implied by equation (1) for this redshift and wavelength range. Therefore, a somewhat more sensitive spectrum of this object could potentially constrain reionization at $z<5.34$.

The $\mathrm{S} / \mathrm{N}$ ratio in the determination of $z_{\text {reion }}$ might be improved by co-adding the spectra of several sources. In the absence of Ly $\alpha$ jungle absorption, the cumulative spectrum would approach the sawtooth template spectrum derived by Haiman, Rees, \& Loeb (1998, their Fig. 1) and would be isotropic across the sky. An alternative signature of reionization should appear at the soft X-ray region of the spectrum $(E \approx 0.1 \mathrm{keV})$, where the continuum optical depth to $\mathrm{H}$ and $\mathrm{He}$ ionization drops below unity. $A X A F$ could detect such a signature in the spectra of only exceptionally bright objects.

In order to enable a measurement of $z_{\text {reion }}$ at redshifts as low as 6 , it is essential that the wavelength coverage of $N G S T$ would extend down to $\sim 7 \lambda_{\beta}=0.7 \mu \mathrm{m}$. Detection of the transmitted flux features requires progressively higher sensitivities as $z_{\text {reion }}$ increases because of the likely increase in the optical depth of the Ly $\alpha$ jungle with redshift. For sudden reionization, the nominal integration time of about $10 \mathrm{hr}$ for a $\sim 10 \mathrm{nJy}$ sensitivity (with $R=100$ and $\mathrm{S} / \mathrm{N}=10$ ) would be sufficient to determine $z_{\text {reion }}$ up to a redshift of $\sim 7$ with a precision of several percent. More gradual reionization would smear the edge of the GP trough. Following the extrapolation of equation (1) to higher redshifts, the required sensitivity needs to be 1 or 2 orders of magnitude higher if $z_{\text {reion }} \sim 8$ or $\sim 9$. Note, however, that if $z_{\text {reion }} \gtrsim 10$, then it will be more easily measurable through the damping of the CMB anisotropies on small angular scales (HL98). Near the planned spectroscopic sensitivity of NGST, most sources are expected to have redshifts lower than $z_{\text {reion }}$. However, suitable highredshift sources could be preselected photometrically in analogy with the UV dropout technique (Steidel et al. 1996) by searching for the expected drop in the flux at the blue edge $\left[\lambda_{\alpha}\left(1+z_{\text {reion }}\right)\right]$ of the Ly $\alpha$ GP trough.

This work was supported in part by the NASA ATP grants NAG 5-3085, NAG 5-7039, and the Harvard Milton fund. We thank Martin Rees for comments, George Rybicki and Mike Shull for advice on the statistics of the Ly $\alpha$ clouds, and Peter Stockman for useful information about NGST.

\section{REFERENCES}

Arons, J., \& Wingert, D. W. 1972, ApJ, 177, 1

Dey, A., Spinrad, H., Stern, D., Graham, J. R., \& Chaffee, F. H. 1998, ApJ, 498, L93,

Fardal, M. A., Giroux, M. L., \& Shull, J. M. 1998, AJ, 115, 2206

Franx, M., Illingworth, G. D., Kelson, D. D., Van Dokkum, P. G., \& Tran, K-V. 1997, ApJ, 486, L75

Gnedin, N. Y., \& Ostriker, J. P. 1997, ApJ, 486, 581

Gunn, J. E., \& Peterson, B. A. 1965, ApJ, 142, 1633

Haiman, Z., \& Loeb, A. 1997, ApJ, 483, 21

—.1998, ApJ, 503, 505 (HL98)
Haiman, Z., Madau, P., \& Loeb, A. 1999, ApJ, 514, 535

Haiman, Z., Rees, M. J., \& Loeb, A. 1997, ApJ, 476, 458 (HRL97)

Hernquist, L., Katz, N., Weinberg, D. H., \& Miralda-Escudé, J. 1996, ApJ, 457, L51

Hu, E. M., Cowie, L. L., \& McMahon, R. G. 1998, ApJ, 502, L99

Loeb, A. 1998, in ASP Conf. Ser. 133, Science with the Next Generation

Space Telescope, ed. E. Smith \& A. Koratkar (San Francisco: ASP), 73

Madau, P. 1995, ApJ, 441, 18

Miralda-Escudé, J. 1998, ApJ, 501, 15

O’Flaherty, K. S., \& Jakobsen, P. 1997, ApJ, 479, 673 
Osterbrock, D.E. 1974, Astrophysics of Gaseous Nebulae (San Francisco: Freeman)

Peebles, P. J. E. 1968, ApJ, 153, 1

Press, W. H., \& Rybicki, G. B. 1993, ApJ, 418, 585

Press, W. H., \& Schechter, P. L. 1974, ApJ, 181, 425

Schneider, D. P., Schmidt, M., \& Gunn, J. E. 1991, AJ, 102, 837

Scott, D., Silk, J., \& White, M. 1995, Science, 268, 829

Shapiro, P. R., \& Giroux, M. L. 1987, ApJ, 321, L107
Shapiro, P. R., Giroux, M. L., \& Kang, H. 1987, in High-Redshift and Primeval Galaxies, ed. J. Bergeron, D. Kunth, B. Rocca-Volmerange, \& J. Tran Thanh Van (Paris: Editions Frontières), 501

Songaila, A. 1997, ApJ, 490, L1

Steidel, C. C., Giavalisco, M., Dickinson, M., \& Adelberger, K. L. 1996, AJ, 112,352

Weymann, R. J., Stern, D., Bunker, A., Spinrad, H., Chaffee, F. H., Thompson, R. I., \& Storrie-Lombardi, L. J. 1998, ApJ, 505, L95 